\title{
P03-2-28 Poster session
}

\section{The role of endogenous chaperone protein RTP4 in Opioid Receptor Heteromer Regulation}

\author{
Wakako Fujita ${ }^{1}$, Mini Yokote ${ }^{2}$, Hiroshi Ueda ${ }^{2}$, Lakshmi A Devi ${ }^{3}$ \\ ${ }^{I}$ Department of Frontier Life Science, Nagasaki University Graduate School of Biomedical Sciences, Japan, \\ ${ }^{2}$ Department of Pharmacology and Therapeutic Innovation, Nagasaki University Graduate School of Biomedical \\ Sciences, Japan, ${ }^{3}$ Department of Pharmacology, Icahn School of Medicine at Mount Sinai, NY, USA
}

A number of studies have revealed that MOPr and DOPr associate to form heteromers in brain regions involved in pain processing and that the levels of these heteromers (MOPr-DOPr) are upregulated following chronic morphine administration under a paradigm that leads to the development of antinociceptive tolerance. However, very little is known about the mechanism underlying MOPr-DOPr upregulation in the brain. A previous study reported that a chaperone, the receptor transporter protein 4 (RTP4), enhanced the cell surface expression of MOPr-DOPr by protecting heteromers from ubiquitination and proteosome-mediated degradation. Here we describe studies to characterize the regulation of RTP4 by opiates. We find that treatment of cells co-expressing MOPr and DOPr with opioid receptor ligands leads to a significant increase in RTP4 mRNA expression. This effect is specific to opioid receptors since ligands targeting other G protein-coupled receptors such as serotonin or dopamine, do not lead to this increase in RTP4 expression. Moreover, chronic morphine administration to mice induces a significant increase in RTP4 expression in distinct brain regions. This effect is specific to RTP4 since chronic morphine administration does not lead to increases in other related chaperones such as other RTPs (1 to 3 ) or receptor expression enhancing proteins (REEPs). Interestingly, in the regions with RTP4 upregulation following chronic morphine administration we observe increases in the levels of MOPr-DOPr and of MOPr with no changes in the levels of DOPr, CB1 cannabinoid or D2 dopamine receptors. Together these results suggest an important role for RTP4 in regulating MOPr-DOPr after long-term opioid treatment. Supported by JSPS KAKENHI grants 16K19214 (to WF). 